\title{
RFID-BASED USERS' LOCALIZATION IN THE CROWDED AREAS
}

\author{
Noor E. Baqir ${ }^{1}$, Mohammed I. Al-Nouman ${ }^{2}$ \\ 1,2 College of Information Engineering, Al-Nahrain University, Baghdad, Iraq \\ \{noor.emad, m.aalnouman $@$ coie-nahrain.edu.iq ${ }^{1,2}$ \\ Received:8/2/2021, Accepted:27/3/2021
}

\begin{abstract}
Managing crowded areas is an extremely and difficult task. Several people might be injured or lost their lives every year because of the mal organization of the crowded areas. There are many regular crowded events where this problem continues and create social and personal upheaval, namely, if not resolved, perhaps destroy the image of the administrators and organizers. One such event is the anniversary pilgrimage Hajj, where Millions visit the holy site Makkah (mecca) in Saudi Arabia. Besides, other regular events attract much more participants. During hajj events, some of the pilgrims get lost and are unable to reach back to their places. Many techniques are used to track users in crowded areas, like Wi-Fi, Mobile networks, and RFID. In this article, we proposed an integrated management model to solve this problem. The proposed model is based on the web system that uses maps and location service, furthermore, use the IoT tools, particularly RFID Reader and Tag to track and monitor persons. This architecture system can be utilized to solve various challenges related to the management of the crowd in general and the management of pilgrims in particular. This model can be adapted also to any crowded event.
\end{abstract}

\section{keywords: Localization, RFID tracking, Crowd management, Cloud computing.}

\section{INTRODUCTION}

Crowd management and tracking are very crucial to successfully organize and conduct big gatherings where there is always a concern of security and safety [1], [2]. through 2001-2016, there were about 7 thousand people lost their life due to stampedes throw the crowded areas [3], [4]. Further, many of the users have their kids or children with them and have lost them in the crowd [5]. So needed to find a way to find people's location in the crowded area. There are many areas where a large crowd of people where gathered, like Hajj (pilgrimage) [6], [7], where a huge gathering of Muslims from different countries gathered at the same time, this event has caused more than 4,000 stampede-related deaths in the last 25 years, the Hajj season becomes more challenging, especially when the entire crowd is the selfsame movements at the selfsame times doing the same thing [3], [8], [9]. Other larger pools like Kumbh Mela, which takes place once every three years in India, likewise witnessed hundreds of stampedes-related deaths recently [9]. plus, the yearly pool of millions of people in Karbala, Iraq in events that happened twice yearly [10]. Basically, any group of people that gathered to do any activities will have the concern for loss people of even got hurt like the concert, football matches, and marathons which held in different countries and attract more than 15000 participants [11]. Several kinds of wireless technology are used in both outdoor and indoor localization which are mainly used to track specific users in congested areas, some of these techniques are using GPS (Global Positioning System) [12], [13] however such a signal may be weak or even not available in some areas [14], [15]. Another approach that using different sensors along with the smartphone to sense a person's activity in real-time can be used as well this is called Smartphone-based Human Activity Recognition (HAR) [14]. Also, WSN (Wireless Sensor Networks) using a BSN (Body Sensor Network) can be used to track the user's activities in indoor areas [16]. Moreover, UWB (Ultra-Wide Band) has been used along with the traditional communication technologies, however hardware implementation of UWB is more expensive than other technology [17]. Additionally, many new technologies can be used to track or localized the users such as Bluetooth (IEEE 802.15), ZigBee, Image-Based Technologies/Cameras 
Based Technology [12], [17]- [20]. A crowd management system must address many issues on a priority basis. In specific, it must be able to track missing persons. Some of the indoor localization technologies and algorithms cannot be used in outdoor localization and vice versa. [17]. In crowded areas there is a problem of data transfer could become a bottleneck on the internet router or repeater, as there are too many users trying to send or receive data at the same time [14], [16] and this the same situation if the cellular network has been used to send the data. So directed to solve the problem using the RFID. It is strongly believed that radio frequency identification can be an ideal personal identification and crowd control solution in crowd areas especially in the indoor or specific routes or paths [16], [21]. The main problem that this paper trying to solve is to localize the person in crowded areas for a specific path or location, the main contribution of this paper is to use the RFID and cloud server to localize the users in an outdoor environment to reduce the number of connected devices to the internet and solve the problem of the bottleneck. The main challenge of the project is how to do this in outdoor environments as the RFID is usually used in indoor areas. The main objectives of the proposed system will be: find the user location in the crowed, Establishing customer service centers in many locations in the crowded area by building a web application provided to the center's employees to track, deliver and retrieve the lost persons to their guardians or owners. To apply this proposed system needed an RFID tag given each person a tag (properties this tag distribute to each user, the price of this is cheaper and can be used again), plus an RFID reader distribute in a particular area (this device is installed and programming one time). furthermore, each reader connects to the wireless router to send the UID of the tag to the database on the cloud. The rest of the paper is organized as follows: section two will be the literature review, section three will introduce the suggested architecture, section four is related to the result and the final section five will be the conclusion.

\section{Related Work}

In this section, a lot of researches on wireless localization of crowded areas will be presented. These researchers have developed and enhanced the most important work in this field. Many researchers are surveying to show the different types of localization in crowded areas like Khudhair, A. A. , et al., they presented a study by doing a survey and compressions between the existing indoor localization technologies. In this paper, they tried to present a survey of existing indoor positioning solutions by classifying various systems and technologies showing the advantages and disadvantages of each one [17]. Additionally,Yamin, M. analyzing different types of crowds and techniques that can be useful for the best prevention of disasters and crowd management. They selected two cases, Kumbh and Hajj, for their study of large crowded events. A special look at the crowded nature of these events. In this operation, the merits of the various technologies and tools that are actually in use or could be considered for use have been examined. In this research, some factors are suggested to analyze and reduce the risk of disasters that happened in crowded areas [3]. As mentioned in the introduction many technologies can be used to track users like using the GPS/GPRS which is used by many researchers like Amro, A., and Q. Nijem who proposed a system that using the combination of GPS and GPRS (General Packet Radio Service) for pilgrims tracking. The tracking information can be accessed by a group leader (guide), this device will be attached in the users' hand (wristbands). In this system, the guide of the group in Hajj by using various portable devices can monitor the assigned group of pilgrims through a web-based application. And then send the users location instantly to the webserver using the 
GPRS packet through the (GSM) Global System for Mobile network, all the information will be stored in a Mobile Object Database (MOD) [5]. More by using the GPRS/GPS, Parveen, Z. and K. Aldhlan built a central control system using Arduino UNO Microcontroller which can help to track down pilgrims that would be missed during the Hajj event. They proposed a system designed in a way to handle both solutions at the same time. In the proposed system, the Hajj site is immediately sent to the web server in the form of a General Packet Radio Service (GPRS) packet through the Global Mobile Phone System (GSM) network upon request [22]. In the same way Lim, S. A. developed a pilot program that uses GPS, GSM modem to track users, the proposed system used the Arduino UNO as a microcontroller for navigation purposes to track the pilgrims during the Hajj and Umrah events using wireless sensor networks (WSN). Through the microcontroller, the GPS will provide users' information in real-time and the location of the users then sends the coordinates to a database through the GSM modem. The main purpose of using the Microcontroller is it is considered as a crossing point between the GPS receiver and GSM modem. The data is sent and received using the SMS (Short Message Service) [23]. The RFID is also can be used to localized the users so that Mohandes, M. , described a model of a system that used passive RFID tags to identify users (pilgrims) in the holy places during the Hajj event. When the RFID card is presented to a handheld reader, the information of the user who has the card will be displayed on the screen of the RFID reader [24]. Furthermore, Ebi, C. , S. Franco-Gomez, and T. Wang proposed a hybrid localization algorithm for accurate internal geolocation, technologies used: Wi-Fi, RFID, and UWB, the proposed system which is written in MATLAB suggest an algorithm to get the data and information from Wi-Fi, RFID, and UWB, and produce significantly more accurate localization method. The system is designed and implemented the hybrid localization algorithm to integrate these different technologies to take advantage of their individualism tracking capabilities [15]. Tufail proposed a state-of-the-art wireless technology to solve these potential problems in crowded areas. They suggested a novel architecture and algorithms using the combination of RFID and WSN to provide monitoring, tracking, and location-based services for the users. This proposed architecture can be utilized to solve various challenges related to the management of the crowd in general and the management of pilgrims in particular [1]. As the data transfer is an issue that the researcher trying to solve but also the data storage and database management can be improved as well as done by Elazhary $\mathrm{H}$. who proposed a normative framework for the distributed management of such crowded events. Within this framework, a global cloud-based server is being used to communicate public information with the pilgrims' mobile application. On the other hand, assigned each campaign on a cloud-based campaign server to communicate its campaign information with its corresponding Hajj applications [7]. From the literature above, we can conclude that many technologies can be used to localize and monitor the users, the most famous is the GPS/GPRS and the RFID. As the GPS/GPRS has some limitations in terms of coverage and data bottleneck. While in the other hand the RFID reader can act as a sink to collect all the information and send it to the cloud to be stored in the database. For that reason, We used the RFID in this paper. However, the proposed work differs from other's work in that our work will use the RFID in the outdoor environment and that is the main challenge and contribution.

\section{SUggested Work}

Radio Frequency Identification (RFID) is an electronic marking technology that permits the identification of a person, a place, or an object automatically and remotely by using an electromagnetic charge/response exchange. exemplary 
applications encompass product labelling for quick checkout at the point of sale, secure auto keys, animal marking, access control of secured facilities, marathon runners, and inventory tracking [24], [25]. The RFID system contains two components [20] [26] the transceiver or RFID reader, and the transponder or RFID tag with a scanning antenna. The RFID reader (transceiver) is a device used to collect information from the (transponder) RFID tag, which is using to track individual persons or objects. The scanning antenna outputs the RF signals in a relatively short range like Radio waves that transfer data from the tag to the reader. Radiofrequency radiation does two things; (in the case of passive RFID tags) providing it with the power needed to operate and providing a means of communicating with the transceiver tag (RFID chip). An RFID tag is consist of a wireless transducer an antenna and an encapsulating material [21]. These tags can be classified depends on how the tag communicates with the reader: passive or active [27]. The functionality of the overall proposed system from interactions within a system architecture which divided into three parts: Hardware Part where the hardware components are used to implement our proposed work which is: Node MCU as a microcontroller, RFID Reader, RFID Tags. The second part will be the Cloud Part where it is used to store all the user information, we used a Host server (000webhost) with database type MySQL. The database is attached to it to create reports and check the user location and status. The final part is the Monitoring Part, where the administrator can see all the activities of the users by retrieving the information saved in the database. To build this part a Web application has been built with embedded Google Maps with some pages to monitor the user activates. Fig. 1 shows the RFID identification and tracking system.

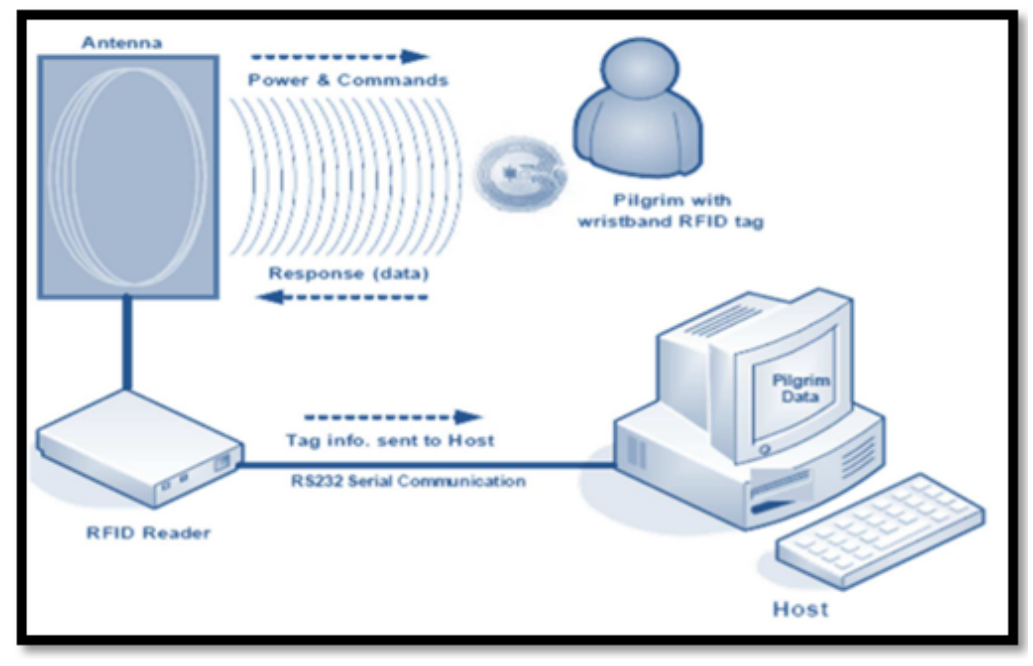

Figure 1: RFID identification and tracking system

\section{SYSTEM IMPLEMENTATION}

The case scenario trying to solve is the area between Baghdad and Karbala in Iraq, this area has activities that happen twice yearly, the area is about (5463 km2)[28], the visitor is from different countries like Pakistan, Iran, India and many others and an age group range from 16 to 60 [10]. the visitor's number can exceed 20 million [10]. The area covered can be seen in Fig. 2. Implement the proposed system throw two phases as shown in Fig. 3 : 


\section{A. Phase One (Pilgrim Record and Information Management Phase):}

In this phase, the RFID reader will distribute for every 100-200 meters, and in some cases will be more or less dense depends on the crowd and also depends on if it is inside or outside the city. Every RFID will have its ID, location (longitude and latitude), and nearby milestone. When the users want to participant in the event, an RFID tag will be given to them, the RFID tag size is the size of a normal badge or ID, When the user comes nearby the RFID reader, the (RFID Reader) reads the (ID) for the user in particularly via a user ID (Reading Process) and then transfer information throw Nude MCU ESP5266 board (Microcontroller Process) and Wi-Fi wireless Router (Transmission Process) to send data to the Cloud server (MySQL and PHP) by wireless (Server Process) to record, manage, and display user information records by a web-based application as shown in Fig. 4.

\section{B. Phase Two (pilgrim monitoring and tracking):}

At this stage, through a reading process, the RFID reader reads pilgrim's UID, then Nude MCU (ESP8266 microcontroller) is used to transfer pilgrim information to 000webhost server through Wi-Fi transmission, at the server (MySQL and PHP) where searches for UID of a particular pilgrim and fetches his data from the database like (name, phone number, gender, Email etc) then the information can be presented on the central screen and also display the location of a pilgrim on the map.

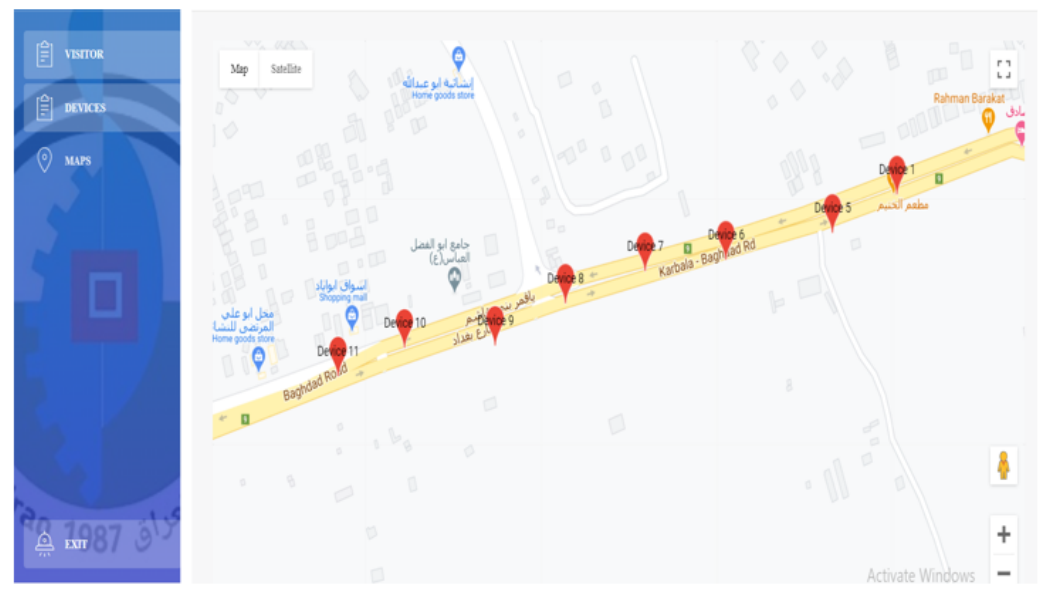

Figure 2: View of area covered

The main objective of this paper is to assist governments to find lost persons quickly and easily. In this paper, RFID is the major hardware component which is using radio waves to capture and read information stored on a tag linked to a person or an object. The tag information can be read from a distance of up to several feet and does not need to be within the reader's direct reader's line of sight to track it. The entire flow of the tracking pilgrim identify is shown in Fig. 5. 


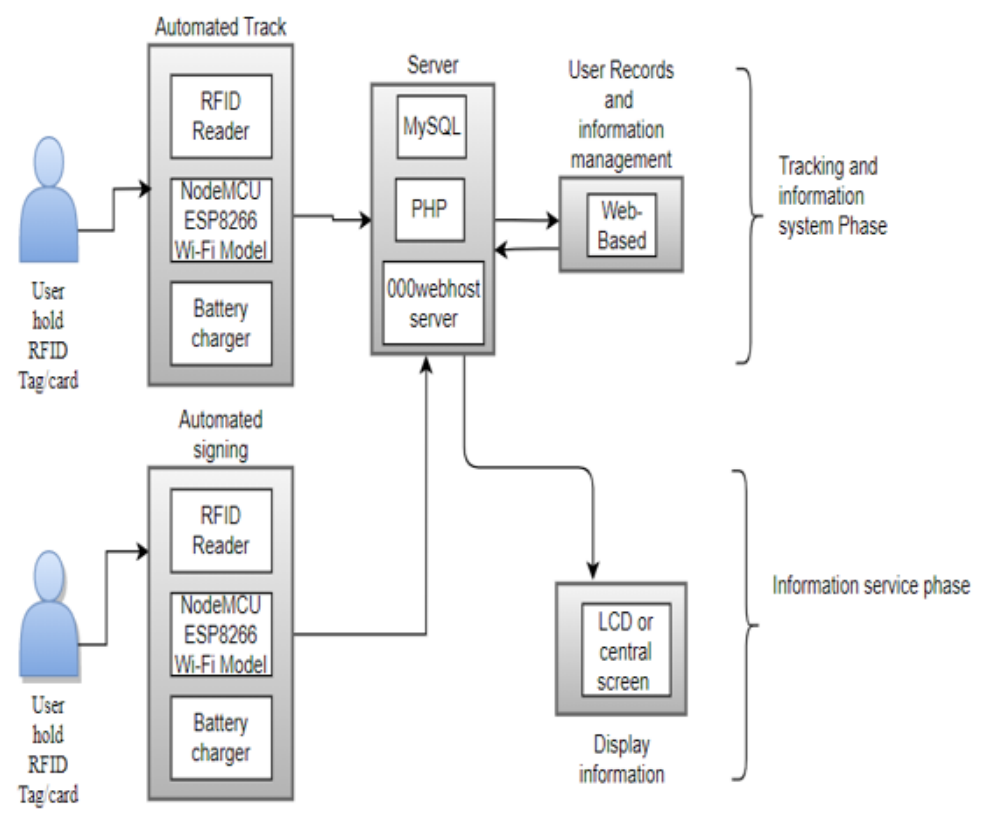

Figure 3: Block diagram for the system model

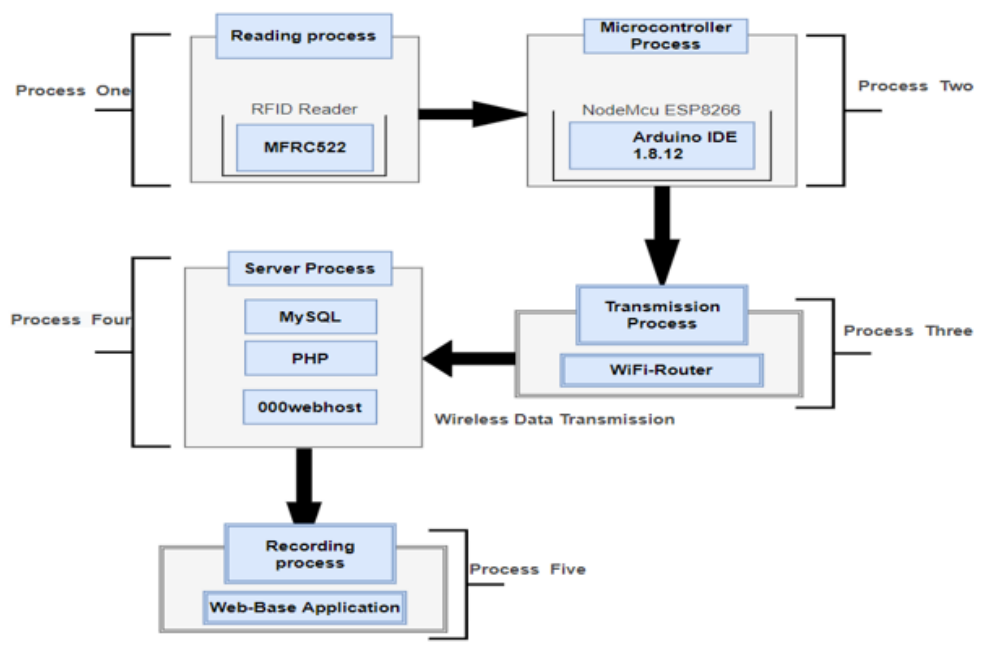

Figure 4: Procedures steps for pilgrims tracking and information management phase

Our proposed work has many parts to achieve its aim, as the information read by the RFID reader and sent to the cloud, an application or web site is needed to read this information, to do so we built a web-based application, which covers all the main operations of service centers dealing with adding, deleting, editing report of found or lost persons. Google maps will be utilized as technology to be used as a web mapping service application provided by Google. High-resolution aerial 
and satellite images for most urban areas over the world have been provided by Google Maps services. Embed Google Maps on the website is configured via utilizes JavaScript Application program interface (API) provided by Google. When the person who holds the card passes from the reader, the reader reads the UID of the tag and sends this UID id to the database on the server. And then the website will localize the users on the map and can track their activities.

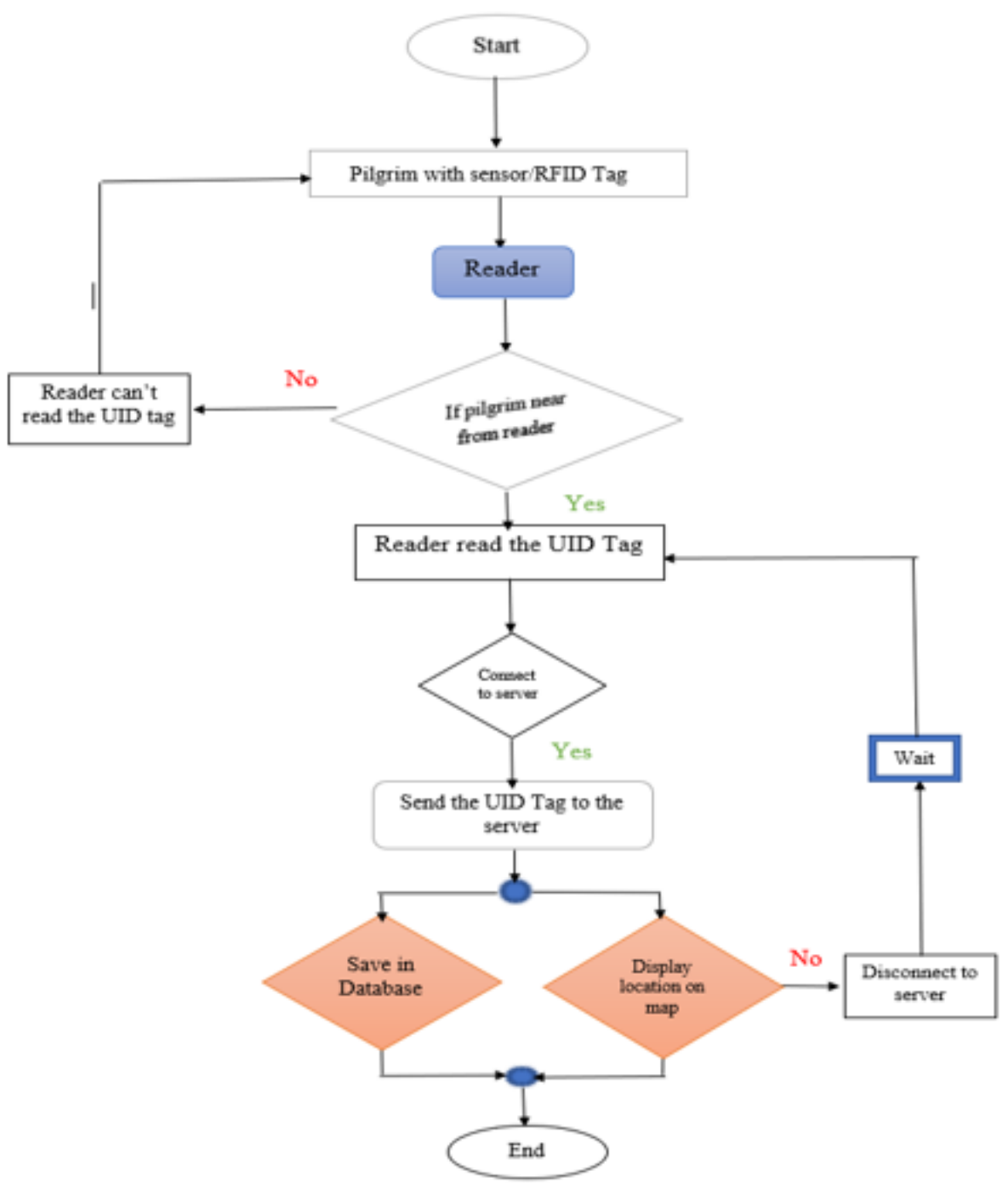

Figure 5: Complete flow of the work

\section{Database Management}

The system is implemented by the MySQL database management system and ADODB. MySQL is the most widely used open-source Relations Database Management system RDBMS that executes as providing multi-user access to several databases. The major factor of using the MySQL database management system is considered compatible with PHP language 
in addition, it has been configured to be used with stored procedures. The method of encapsulating repeated tasks is called a stored procedure which is used to isolate users from the data table and improve performance due to it reduces network traffic. The database consists of three tables: users table, devices table, and tracking table. The first table includes basic account information of the user, while the second table contains information about the RFID reader and the last table contains track location coordinates of all agents. Each table contains several records with various types of data as characterized in the following segments, Fig. 6 shows a diagram of the Entity-Relationship database. Table I shows the main table that contains basic account information.

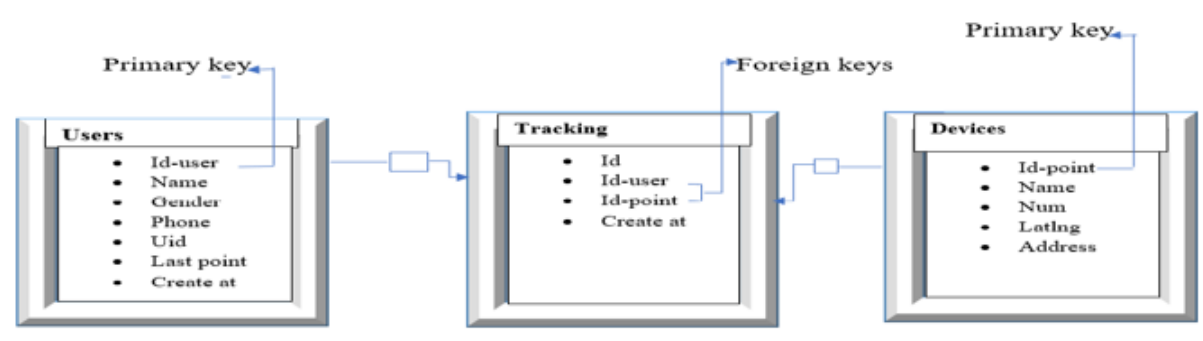

Figure 6: Entity- relationship database

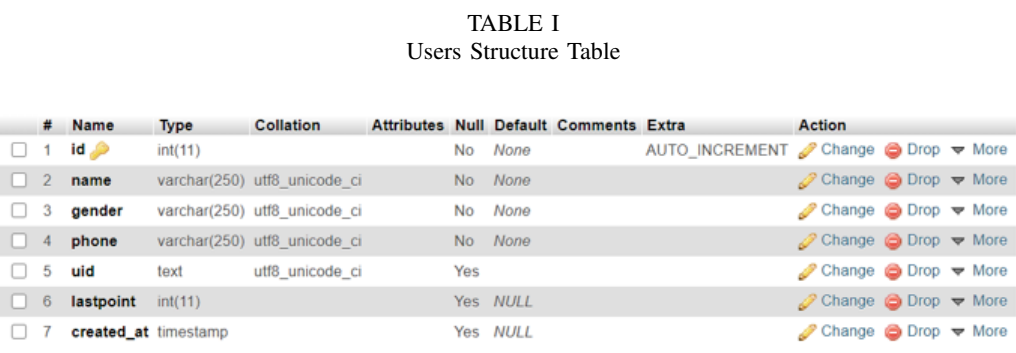

Table II shows the Devices table which is used to keep information associated with RFID reader. This Table contains ID number of reader, name of devices, latitude and longitude of coordinates that consider decimal type.

\begin{tabular}{|c|c|c|c|c|c|c|c|c|}
\hline & & & & Device & $\begin{array}{l}\text { TAB } \\
\text { e Str }\end{array}$ & $\begin{array}{l}\text { BLE II } \\
\text { ructure Table }\end{array}$ & & \\
\hline$\#$ & Name & Type & Collation & Attributes & Null & Default Comments & Extra & Action \\
\hline$\square 1$ & id $P$ & $\operatorname{int}(11)$ & & & № & None & AUTO_INCREMENT & $\Rightarrow$ Change $\theta$ Drop $\nabla$ More \\
\hline$\square 2$ & ID-number & $\operatorname{varchar}(250)$ & utf8_unicode_ci & & No & None & & $\partial$ Change $\theta$ Drop $\nabla$ More \\
\hline$\square 3$ & name of device & varchar(250) & utfi_unicode_ci & & No & None & & Change $\theta$ Drop $\nabla$ More \\
\hline D 4 & lating & $\operatorname{varchar}(250)$ & utf8_unicode_ci & & Yes & NULL & & $\partial$ Change $\odot$ Drop $\nabla$ More \\
\hline$\square 5$ & address & varchar(250) & utf8_unicode_ci & & Yes & NULL & & $\partial$ Change $\theta$ Drop $\nabla$ More \\
\hline
\end{tabular}

Table III is designed to record and store the pilgrim's or user position in real-time and the history of the user location. The foreign key has stored to link this table to the device table. And Table IV illustrates the structure of the tracking table. 


TABLE III
Track Structure Table
\# Name Type Collation Attributes Null Default Comments Extra

\section{RESULTS}

This paper introduces an indoor RFID localization system capable of locating hundreds of people using five RFID receiving stations within a kilometer area, each user would have an RFID enable Tag or card. We tested them for two hours and then checked the site on the cloud server to monitor the users' location, the scenario we applied is in the road Baghdad- Karbala in Iraq using an RFID card that can be wearable at any time and will be provided to the user for every person who wants to join the event. The information stored in the cloud can be retrieved by the person who has the access to the website. To check the user last locations the in-charged person will enter the user's ID and all the information will be displayed on the map and in a table as can be seen in Fig. 7 and Table IV.
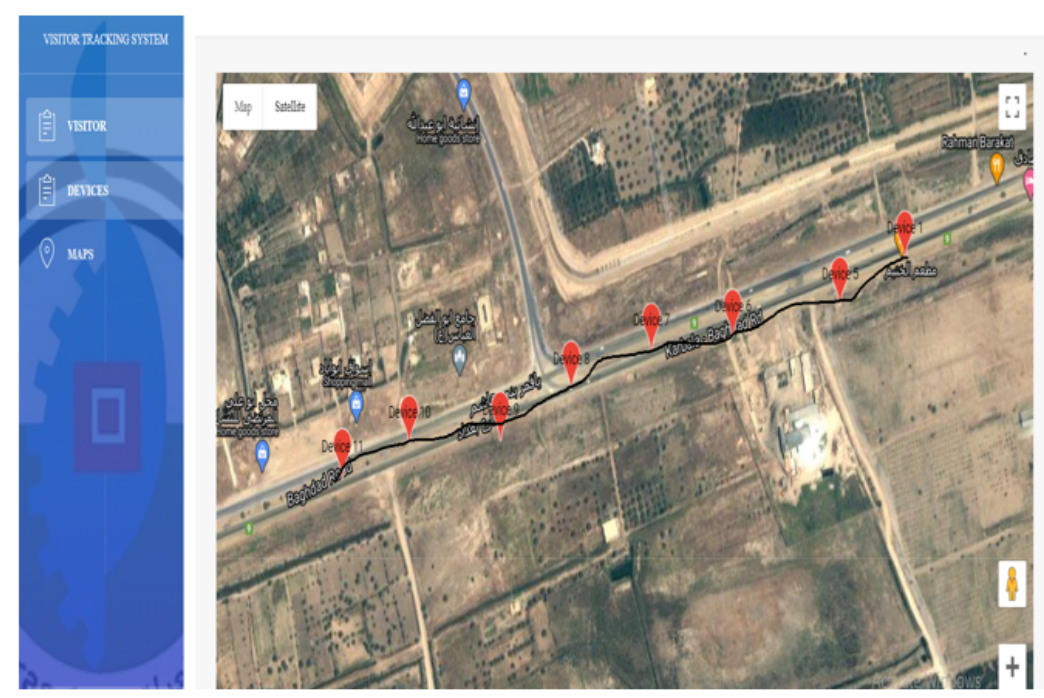

Figure 7: Satellite view of the device location 


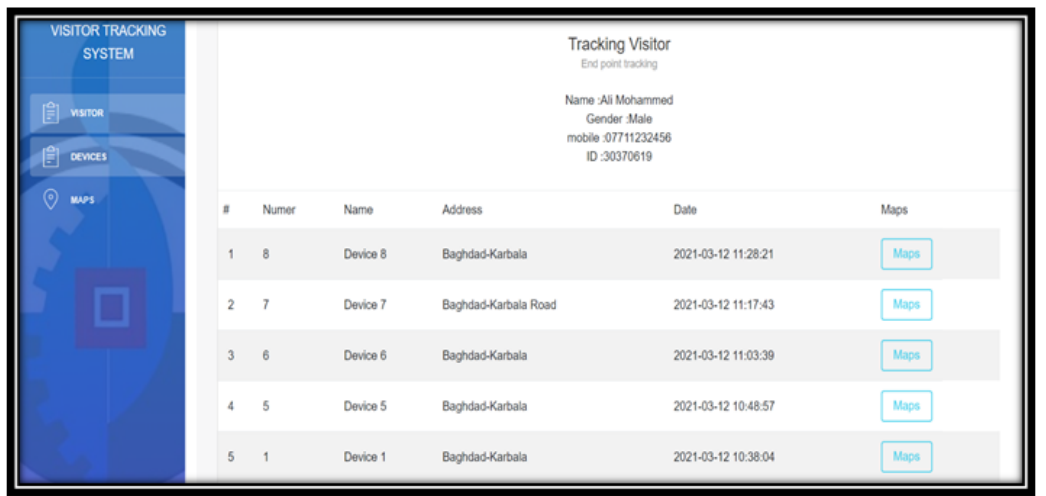

\section{CONClusion}

The management of crowded areas is complex and very challenging. Where several people may be lost their lives because of the unorganized crowded areas. There are several methods to locate users' locations for outdoor and indoor environments, however, some of these technologies have some limitations regarding data transmission. In the proposed system we suggest the RFID to localize the in a specific path, the user will wear the RFID tag and the RFID readers that distributed along the path will read the user's location and send this information to the cloud-based database. Additionally, a web application is developed to track users in crowded areas. Authorized persons can check the database to retrieve the history of the user's location and path. We tested the proposed system in different scenarios and the user's information has been sent and retrieved successfully when needed. 


\section{REFERENCES}

[1] A. Tufail, "Pilgrim Tracking and Location Based Services Using RFID and Wireless Sensor Networks" , IJCSNS, Vol. 18, No. 6, p. 112, 2018.

[2] A. Khan, J. Ali Shah, K. Kadir, W. Albattah, and F. Khan, "Crowd Monitoring and Localization Using Deep Convolutional Neural Network: A Review" , Applied Sciences, Vol. 10, No. 14, p. 4781, 2020.

[3] M. Yamin, "Managing Crowds with Technology: Cases of Hajj and Kumbh Mela" , International journal of information technology, Vol. 11, No. 2, pp. 229-237, 2019.

[4] M. Rodrigues Leal Moitinho de Almeida, "Human Stampedes: A Scoping Review" , 2016.

[5] A. Amro and Q. Nijem, "Pilgrims Hajj Tracking System (e-Mutawwif) ", Contemporary Engineering Sciences, Vol. 5, No. 9, pp. 437-446, 2012.

[6] A. M. Alsubhy, A. A. Abi Sen, B. A. Alahmadi, N. M. Bahbouh, and H. A. Abi Sen, "A Model for Tracking People and Property in Crowds", in 2020 7th International Conference on Computing for Sustainable Global Development (INDIACom), IEEE, pp. 244-248, 2020.

[7] H. Elazhary, "Cloud-Based Context-Aware Mobile Applications and Framework for Hajj and Umrah Management" , Int. J. Comput. Trends Technol. , Vol. 47, No. 2, pp. 106-115, 2017.

[8] M. Yamin, A. M. Basahel, and A. A. Abi Sen, "Managing Crowds with Wireless and Mobile Technologies", Wireless Communications and Mobile Computing, Vol. 2018, 2018.

[9] M. Yamin, "Health Management in Crowded Events: Hajj and Kumbh" , BVICA M's International Journal of Information Technology, Vol. 7, No. 1, p. 791, 2015.

[10] U. M. Husein, "A Phenomenological Study of Arbaeen Foot Pilgrimage in Iraq" , Tourism Management Perspectives, Vol. 26, pp. 9-19, 2018.

[11] A. Burfoot, "The History of The Marathon", Sports Medicine, Vol. 37, No. 4-5, pp. 284-287, 2007.

[12] A. Mustafa, M. I. al-Nouman, and O. A. Awad, "Cloud-Based Vehicle Tracking System" , Iraqi Journal of Information and communication technology, Vol. 2, No. 4, pp. 21-30, 2019.

[13] O. A. Abdullah, M. Aal-nouman, and A. Al-joudi, "Indoor WLAN Localization via Adaptive Lasso Bayesian Inference and Convex Optimization" , Cogent Engineering, Vol. 7, No. 1, p. 1813677, 2020.

[14] M. O. Gani et al. , "An Approach to Localization in Crowded Area," , in 2016 IEEE 18th International Conference on e-Health Networking, Applications and Services (Healthcom), IEEE, pp. 1-6, 2016.

[15] C. Ebi, S. Franco-Gomez, and T. Wang, "Hybrid Wi-Fi Localization Using RFID and UWB Sensors" , 2015.

[16] A. Boudhir, M. Bouhorma, M. Benahmed, and S. Elbrak, "A Real Time Health Control and Localization of Pilgrims in El Hajj: BSN Application" , Journal of Theoretical \& Applied Information Technology, Vol. 49, No. 2, 2013.

[17] A. A. Khudhair, S. Q. Jabbar, M. Q. Sulttan, and D. Wang, "Wireless Indoor Localization Systems and Techniques: Survey and Comparative Study" , Indonesian Journal of Electrical Engineering and Computer Science, Vol. 3, No. 2, pp. 392-409, 2016.

[18] M. Meingast, S. Oh, and S. Sastry, "Automatic Camera Network Localization Using Object Image Tracks" , IEEE 11th International Conference on Computer Vision, IEEE, pp. 1-8, 2007.

[19] D. Stojanovic and N. Stojanovic, "Indoor Localization and Tracking: Methods, Technologies and Research Challenges" , Facta Universitatis, Series: Automatic Control and Robotics, Vol. 13, No. 1, pp. 57-72, 2014.

[20] R. D. Al-kafaji, S. K. Gharghan, and S. Q. Mahdi, "Localization Techniques for Blind People in Outdoor/Indoor Environments" , in IOP Conference Series: Materials Science and Engineering, Vol. 745, No. 1: IOP Publishing, p. 012103, 2020.

[21] M. Mohandes, "An RFID-Based Pilgrim Identification System (A Pilot Study) " , 11th International Conference on Optimization of Electrical and Electronic Equipment, IEEE, pp. 107-112, 2008.

[22] Z. Parveen and K. Aldhlan, "Missing Pilgrims Tracking System Using GPS, GSM and Arduino Microcontroller" , in International Conference on Recent Advances in Computer Systems, Atlantis Press, 2015.

[23] S. A. Lim, "People Tracking System Using Global Positioning System and Global System for Mobile Communication" , Universiti Teknologi Malaysia, 2013.

[24] M. Mohandes, "RFID-Based System for Pilgrims Identification \& Tracking", J. Appl. Comput. Electromagn. Soc. (ACES), Vol. 25, No. 3, pp. $273-282,2010$.

[25] M. J. Deka, J. Joshi, N. Sinha, A. Tyagi, A. Kushal, and A. Jain, "Indoor and Outdoor Position Identification Using RFID" , in 2016 International Conference on Recent Advances and Innovations in Engineering (ICRAIE), IEEE, pp. 1-7, 2016.

[26] A. Ortega Piris, E. Diaz Ruiz, C. PÃ@rez Labajos, and A. Navarro Morales, "Implementation of A RFID System on Ships for Passenger and Crew Location" , in Maritime Transport VIII: proceedings of the 8th International Conference on Maritime Transport: Technology, Innovation and Research: Maritime Transport'20, Universitat Politecnica de Catalunya. Departament de Ciencia i Enginyeria, pp. 311-329, 2020.

[27] Y. Berdaliyev and A. P. James, "RFID-Cloud Smart Cart System", in 2016 International Conference on Advances in Computing, Communications and Informatics (ICACCI), IEEE, pp. 2346-2352, 2016.

[28] Y. R. Ensaif and M. A. Al-Anbari, "Landfill Site Selection in Karbala Governorate, Iraq" , Journal of Engineering and Sustainable Development, Vol. 22, No. 6, pp. 30-42, 2018. 